\title{
Catalogue of Rose Gall, Herb Gall, and Inquiline Gall Wasps (Hymenoptera: Cynipidae) of the United States, Canada and Mexico
}

\author{
Louis F. Nastasi ${ }^{\ddagger}$, Andrew R. Deans ${ }^{\ddagger}$ \\ ‡ Frost Entomological Museum, Penn State University, University Park, United States of America
}

Corresponding author: Louis F. Nastasi (Ifnastasi@gmail.com), Andrew R. Deans (adeans@psu.edu)

Academic editor: Matthew Yoder

Received: 11 May 2021 | Accepted: 15 Jul 2021 | Published: 24 Aug 2021

Citation: Nastasi LF, Deans AR (2021) Catalogue of Rose Gall, Herb Gall, and Inquiline Gall Wasps

(Hymenoptera: Cynipidae) of the United States, Canada and Mexico. Biodiversity Data Journal 9: e68558.

https://doi.org/10.3897/BDJ.9.e68558

\begin{abstract}
Background

Cynipidae (Hymenoptera: Cynipoidea) is a diverse group of wasps, many of which are capable of inducing plants to make galls, novel structures that protect and nourish the wasps' larvae. Other cynipids, especially those species in Ceroptresini and Synergini, are understood to be usurpers of galls made by other cynipids. The North American cynipid fauna has not been fully catalogued since 1979, but there is renewed interest in revising the taxonomy and in doing research that sheds light on the mechanisms of gall induction, the evolution of this life history, and their ecological interactions more broadly. Significant taxonomic changes have impacted the group since 1979, thereby warranting a new catalogue.
\end{abstract}

\section{New information}

The current state of knowledge of species classified in Aulacideini, Ceroptresini, Diastrophini, Diplolepidini, Phanacidini and Synergini in the United States, Canada, and 
Mexico is summarised in catalogue format. We report 323 names, including 170 valid species of rose gall wasps, herb gall wasps, and inquiline gall wasps, classified in 12 genera, from the United States, Canada, and Mexico. Current taxonomic status, distribution, host associations, and vernacular names are listed for each species. The catalogue also includes the original description of galls for many species of gall-inducer, as well as atomised characterisations of different gall traits as key-value pairs. For most galling species without existing vernacular names, new vernacular names are proposed.

\section{Keywords}

biodiversity, checklist, Aulacideini, Ceroptresini, Diastrophini, Diplolepidini, Phanacidini, Synergini

\section{Introduction}

The hymenopteran family Cynipidae constitutes a diverse assemblage of gall-forming and inquilinous wasps, which occur worldwide. While the galls induced by these insects are often conspicuous and charismatic (see Fig. 1), the North American fauna lacks robust classification and accessible diagnostic tools. The group has received moderate phylogenetic attention in recent years, but revisionary taxonomy remains uneven. While the higher taxonomy of the family is uncertain, species that induce galls or act as inquilines are well-distinguished from other Cynipoidea, both in terms of their current taxonomy and natural history.
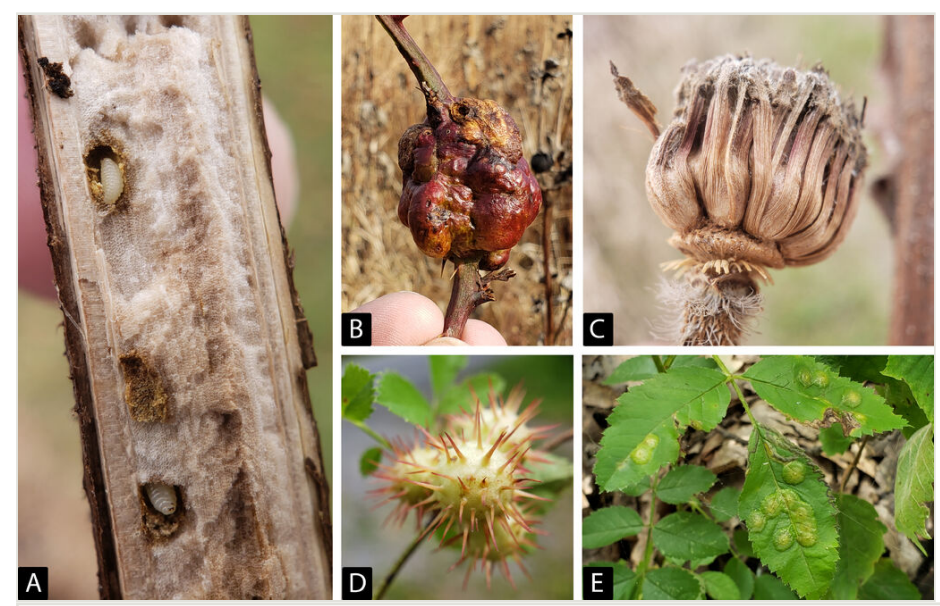

Figure 1. doi

Examples of galls induced by some of the species treated in this catalogue. Photos are not at the same scale. A. Antistrophus jeanae Tooker \& Hanks, 2004 inside Silphium perfoliatum L. stems; B. Diatrophus nebulosus (Osten Sacken, 1861) on Rubus sp. stem; C. Antistrophus laciniatus Gillette, 1891 galls on disc flowers of Silphium laciniatum L.; D. Diplolepis polita (Ashmead, 1890) galls on leaves of Rosa sp.; E. Diplolepis rosaefolii (Cockerell, 1889) galls in leaves of Rosa sp. Photos by Andrew R. Deans 
The present work catalogues the species of tribes Aulacideini, Ceroptresini, Diastrophini, Diplolepidini, Phanacidini and Synergini that are known from Canada, the United States, and Mexico. The higher taxonomy at the tribe level employed herein follows that of Buffington et al. (2020) and Ronquist et al. (2015). Cynipini, which is by far the most species-rich of the family's tribes, is excluded from the present work, primarily due to the existence of many taxonomic works in press or in preparation. While Cynipini is the most diverse of the family's tribes, the herb gallers (Aulacideini, Diastrophini, and Phanacidini), rose gallers (Diplolepidini), and inquilines (Ceroptresini and Synergini) comprise a significant portion of the family's diversity and, therefore, are worthy of their own treatment.

The most recent previous catalogues of the family are Weld (1957), Weld (1959), Weld (1960), Burks (1979), and a database contributed to the Hymenoptera Online database by Johan Liljeblad (Johnson et al. 2019), but many changes to the taxonomy of Cynipidae warrant the development of a new catalogue to reflect recent improved knowledge.

\section{Materials and methods}

Data were compiled using the Darwin Core data standard (Wieczorek et al. 2012) and the Darwin Core Extensions for taxon description, species distribution, and vernacular names. An initial list of cynipid species was obtained by examining the Cynipidae dataset in the Hymenoptera Online database (Johnson et al. 2019). Existing literature relating to the Cynipidae of North America was reviewed in order to generate records relating to the taxonomy, distribution, and biology of cynipid wasps. Data from the community science platform iNaturalist (2021) were also used in order to substantiate locality records and provide the most complete possible distribution for the covered species. iNaturalist observations used in this manner were personally examined by the authors and have been verified to a reasonable degree of certainty to properly represent the organisms contained in the records. Vernacular names were assigned for gall-inducing species that have not yet been assigned one.

Gall descriptions from the literature are provided verbatim for many species. These descriptions were also atomised into individual traits and presented as key-value pairs, using a controlled vocabulary for gall terminology (Deans et al. 2021; included also in the Darwin Core Archive version of this data set). Host associations for numerous Synergini are provided verbatim from the given references (see Suppl. material 1). All association data were indexed by the Global Biotic Interactions resource (GloBl; Poelen et al. 2014).

\section{Data resources}

The catalogue of Aulacideini, Ceroptresini, Diastrophini, Diplolepidini, Phanacidini, and Synergini of the United States, Canada, and Mexico is provided as Suppl. material 1 (Darwin Core Archive) and provides 322 species-level and subspecific names including synonyms. We recognise 170 valid species. The taxonomy of many of these species needs revision, and the intention is to issue subsequent versions of this catalogue to reflect 
substantial changes as they are made in the future. A checklist, which summarises the data set in the Darwin Core Archive, including nomenclature, notes on biology and geographic distribution, is provided below.

Initial data were drawn from the Hymenoptera Online Database (Norm Johnson; Johan Liljeblad). We also used occurrence data from iNaturalist, when we could confidently verify the species' identity, to further establish or even expand the distributions for many species. Botanical taxonomic names were matched to names in the Taxonomic Name Resolution Service.

The Aulacideini, Ceroptresini, Diastrophini, Diplolepidini, Phanacidini, and Synergini of the United States, Canada, and Mexico

\section{Family Cynipidae}

\section{Tribe Aulacideini}

Genus Antistrophus Walsh, 1869

Antistrophus bicolor Gillette, 1891

Feeds on: May induce galls on Silphium integrifolium Michx., but claim is unsubstantiated

Distribution: United States: Illinois

Antistrophus chrysothamni (Beutenmüller, 1908)

Nomenclature:

Aulax chrysothamni Beutenmüller, 1908

Feeds on: Induces galls on Chrysothamnus Nutt.

Distribution: United States: Arizona

Antistrophus jeanae Tooker \& Hanks, 2004

Feeds on: Induces galls on Silphium perfoliatum L.

Distribution: United States: Illinois 
Antistrophus laciniatus Gillette, 1891

Feeds on: Induces galls on Silphium laciniatum L.

Distribution: United States: Illinois, Kansas, Texas, Wisconsin

Antistrophus lygodesmiaepisum Walsh, 1869

Nomenclature:

Antistrophus pisum Ashmead, 1885 | Ascepiadiphila stephanotidis Ashmead, 1897

Feeds on: Induces galls on Lygodesmia juncea D. Don ex Hook

Distribution: United States: Colorado, lowa, Kansas, Missouri, Nebraska, Oregon, South Dakota, Texas; Canada: Alberta, Saskatchewan

Antistrophus meganae Tooker \& Hanks, 2004

Feeds on: Induces galls on Silphium terebinthinaceum Jacq.

Distribution: United States: Illinois

Antistrophus microseris (McCracken \& Egbert, 1922)

Nomenclature:

Aylax microseris McCracken \& Egbert, 1922

Feeds on: Induces galls on Microseris D. Don

Distribution: United States: California

Antistrophus minor Gillette, 1891

Nomenclature:

Aulax Gillettei Kieffer, 1902

Feeds on: Induces galls on Silphium laciniatum L. and possibly other Silphium

Distribution: United States: Illinois

Antistrophus rufus Gillette, 1891

Feeds on: Induces galls on Silphium laciniatum L.

Distribution: United States: Illinois, Kansas 
Antistrophus silphii Gillette, 1891

Nomenclature:

Antistrophus leavenworthi Bassett, 1900

Feeds on: Induces galls on Silphium integrifolium Michx. and S. perfoliatum L.

Distribution: United States: Georgia, Illinois, Indiana, lowa, Kansas, Missouri, Nebraska, Virginia, Wisconsin

Genus Aulacidea Ashmead, 1897

Aulacidea abdita Kinsey, 1920

Feeds on: Induces galls on Lactuca canadensis L. and possibly additional Lactuca L. species

Distribution: Canada: Québec

\section{Aulacidea acroptilonica Tyurebaev, 1972}

Feeds on: Induces galls on Acroptilon repens (L.) DC.

Distribution: United States: Colorado, Montana, Wyoming

\section{Aulacidea ambrosiaecola (Ashmead, 1896)}

Nomenclature:

Aulax ambrosiaecola Ashmead, 1896

Feeds on: May induce galls on Ambrosia L. sp., but claim is unverified

Distribution: United States: Missouri

\section{Aulacidea annulata Kinsey, 1920}

Feeds on: Induces galls on Lactuca L. sp. or possibly Prenanthes

Distribution: United States: Massachusetts, Nebraska

\section{Aulacidea harringtoni (Ashmead, 1887)}

\section{Nomenclature:}

Aulax harringtoni Ashmead, 1887 | Aulax bicolor Gillette, 1891 | Aulax mulgediicola Ashmead, 1896 | Aulax cavicola Ashmead, 1896

Feeds on: Induces galls on Lactuca floridana L. 
Distribution: United States: Connecticut, District of Columbia, Illinois, Massachusetts, Missouri, North Carolina, New Jersey, New York, Oklahoma; Canada: Ontario

Aulacidea nabali (Brodie, 1892)

Nomenclature:

Aulax nabali Brodie, 1892

Feeds on: Induces galls on Nabalus altissimus (L.) Hook

Distribution: United States: Massachusetts, New Jersey, New York; Canada: Ontario

\section{Aulacidea pilosellae (Kieffer, 1901)}

Nomenclature:

Aulax beijerinckii Kieffer, 1899 | Aulax pilosellae Kieffer, 1901

Feeds on: Induces galls on Pilosella Hill spp.

Distribution: United States (Possibly introduced)

Aulacidea podagrae (Bassett, 1890)

Nomenclature:

Aulax podagrae Bassett, 1890

Feeds on: Induces galls on Lactuca canadensis L.

Distribution: United States: District of Columbia, Illinois, Kansas, New York, Ohio, Pennsylvania, Virginia

\section{Aulacidea subterminalis Niblett, 1946}

Feeds on: Induces galls on Hieracium L. spp.

Distribution: United States (Possibly introduced); Canada: British Columbia (Introduced)

\section{Aulacidea tumida (Bassett, 1890)}

Nomenclature:

Aulax tumidus Bassett, 1890 | Aulax sonchicola Ashmead, 1896 | Aulacidea solidaginis Girault, 1903

Feeds on: Induces galls on Lactuca canadensis L.

Distribution: United States: Massachusetts, New Hampshire, Virginia 
Genus Liposthenes Förster, 1869

Liposthenes glechomae (Linnaeus, 1758)

Nomenclature:

Cynips Glechomae Linnaeus, 1758 | Diastrophus similis Bassett, 1881 | Aulax Latreillei Kieffer, 1898

Feeds on: Induces galls on Glechoma hederacea L.

Distribution: United States: Connecticut, Delaware, lowa, Illinois, Indiana, Kansas, Massachusetts, Maryland, Michigan, Minnesota, Nebraska, New Jersey, New York, Ohio, Pennsylvania, Rhode Island, Vermont

\section{Tribe Ceroptresini}

Genus Buffingtonella Lobato-Vila \& Pujade-Villar, 2019

Buffingtonella politus (Ashmead, 1896)

Nomenclature:

Ceroptres politus Ashmead, 1896

Feeds on: Host unknown but presumed to be inquilinous

Distribution: United States: Virginia

\section{Genus Ceroptres Hartig, 1840}

\section{Ceroptres catesbaei Ashmead, 1885}

Feeds on: Inquiline of: galls of Callirhytis quercuscatesbaei Ashmead, 1881 on Quercus laevis Walter

Distribution: United States: Florida

\section{Ceroptres confertus (McCracken \& Egbert, 1922)}

Nomenclature:

Periclistus confertus McCracken \& Egbert, 1922

Feeds on: Inquiline of: galls of Andricus confertus McCracken \& Egbert, 1922 on Quercus lobata Née

Distribution: United States: California 


\section{Ceroptres cornigera Melika \& Buss, 2002}

Feeds on: Inquiline of: galls of Callirhytis quercuscornigera (Osten Sacken 1865) on Quercus palustris Münchh.

Distribution: United States: Kentucky

\section{Ceroptres ensiger (Walsh, 1864)}

\section{Nomenclature:}

Amblynotus ensiger Walsh, 1864

Feeds on: Inquiline of: galls of Andricus quercuspetiolicola (Bassett, 1863) on Quercus bicolor Willd.

Distribution: United States: Illinois

\section{Ceroptres frondosae Ashmead, 1896}

Feeds on: Inquiline of Andricus quercusfrondosus (Bassett, 1865) [as Cynips ? quercusfrondosa] on Quercus prinoides Willd.

Distribution: United States: Missouri

\section{Ceroptres junquerasi Lobato-Vila \& Pujade-Villar, 2019}

Feeds on: Inquiline of: galls of Dros perlentum Kinsey, 1937 on Quercus obtusata Humb. and Bonpl. and Quercus laeta Liebm.; galls of Andricus sphaericus PujadeVillar, 2016 on Quercus rugosa Née and Quercus laeta Liebm.; galls of Andricus georgei Pujade-Villar, 2011 on Quercus candicans Née; galls of Neuroterus eugeros Pujade-Villar, 2018 on Quercus laeta Liebm.; galls of Atrusca pictor (Kinsey, 1936) on Quercus sp.; galls of Atrusca sp. on Quercus obtusata Humb. and Bonpl.; unknown galls on Quercus acutifolia Née; unknown galls on Quercus laeta Liebm.; galls of Melikaiella amphibolensis Pujade-Villar, 2014 on Quercus castanea Née; unknown leaf galls of Neuroterus on Quercus laeta Liebm.

Distribution: Mexico: Ciudad de México, Jalisco, México, Michoacán de Ocampo, Morelos, Puebla

\section{Ceroptres lanigerae Ashmead, 1885}

Feeds on: Inquiline of: galls of Andricus quercuslanigera (Ashmead, 1881) on Quercus minima (Sarg.) Small, Quercus oleoides Schltdl. \& Cham., and Quercus virginiana Mill. (= Q. geminata Small)

Distribution: United States: Florida 


\section{Ceroptres lenis Lobato-Vila \& Pujade-Villar, 2019}

Feeds on: Inquiline of: unknown galls on Quercus laeta Liebm.

Distribution: Mexico: Ciudad de México

\section{Ceroptres mexicanus Lobato-Vila \& Pujade-Villar, 2019}

Feeds on: Inquiline of: unknown spherical leaf galls on Quercus sp.

Distribution: Mexico: México

\section{Ceroptres minutissimi Ashmead, 1885}

Feeds on: Inquiline of: galls of Neuroterus quercusminutissimus (Ashmead, 1885) on Quercus virginiana Mill.; undetermined, non-deciduous galls on leaves of Quercus rugosa Née

Distribution: United States: Florida; Mexico: Ciudad de México

\section{Ceroptres montensis Weld, 1957}

Feeds on: Inquiline of: galls of Andricus reniformis McCracken \& Egbert, 1922 on Quercus vaccinifolia Kell.; galls of Disholcaspis truckeensis (Ashmead, 1896) on Quercus chrysolepis Liebm.

Distribution: United States: California, Oregon

\section{Ceroptres nigricus Lobato-Vila \& Pujade-Villar, 2019}

Feeds on: Inquiline of: undetermined galls on Quercus acutifolia Née; undetermined twig galls on Quercus sp.

Distribution: Mexico: México, Michoacán de Ocampo

\section{Ceroptres petiolicola (Osten Sacken, 1861)}

Nomenclature:

Amblynotus ? petiolicola Osten Sacken, 1861

Feeds on: Inquiline of: galls of Andricus quercuspetiolicola (Bassett, 1863) on Quercus michauxii Nuttall (= Q. prinus auctores not L., = Q. prinus var. michauxii (Nutt.) Chapman 1860); galls of Neuroterus quercusbatatus (Fitch, 1859) [unverified]

Distribution: United States: District of Columbia; Canada: Québec 


\section{Ceroptres pisum (Osten Sacken, 1861)}

Nomenclature:

Sarothrus ? pisum Osten Sacken, 1861

Feeds on: Inquiline of: galls of Acraspis pezomachoides (Osten Sacken, 1862) on Quercus alba L. [unverified]

Distribution: United States: District of Columbia

\section{Ceroptres quadratifacies Lobato-Vila \& Pujade-Villar, 2019}

Feeds on: Inquiline of: galls of Atrusca sp. on Quercus microphylla Née; galls of Sphaeroteras nr. pulchripennis on Quercus microphylla Née

Distribution: Mexico: Aguascalientes

\section{Ceroptres quercusobtusilobae (Karsch, 1880)}

\section{Nomenclature:}

Diplolepis q. obtusilobae Karsch, 1880 | Cynips obtusilobae (Karsch, 1880) | Neuroterus quercusobtusilobae (Karsch, 1880) | Ceroptres obtusilobensis (Karsch, 1880)

Feeds on: Inquiline of: undetermined woody, tuberous galls on Quercus stellata Wengenh. (= Q. obtusiloba Michx.)

Distribution: United States: Texas

\section{Ceroptres rufiventris Ashmead, 1896}

Feeds on: Inquiline of: galls of Amphibolips quercusostensackenii (Bassett, 1863) on Quercus coccinea Müenchh. and Quercus palustris Müenchh.

Distribution: United States: Missouri

\section{Ceroptres snellingi Lyon, 1996}

\section{Nomenclature:}

Ceropteres [sic] snellingi Lyon, 1996

Feeds on: Inquiline of: galls of Andricus flocculentus Lyon, 1996 on Quercus pungens Liebm.; galls of Andricus sphaericus Pujade-Villar, 2016 on Quercus mexicana Bonpl.; galls of Andricus sphaericus Pujade-Villar, 2016 on Quercus rugosa Née; galls of Andricus sp. on leaves of Quercus cf chihuahuensis Trel.; galls of Andricus sp. on leaves of Quercus cf eduardi Trel.; undetermined leaf petiole galls on Q. potosina Trel.; galls of Andricus sp. (georgei group) on Quercus sp. 
Distribution: United States: Texas; Mexico: Aguascalientes, Ciudad de México, México, Nuevo León, Zacatecas

\section{Tribe Diastrophini}

\section{Genus Diastrophus Hartig, 1840}

\section{Diastrophus austrior Kinsey, 1922}

\section{Nomenclature:}

Diastrophus kincaidii var. austrior Kinsey, 1922

Feeds on: Induces galls on Rubus parviflorus Nutt. and R. nutkanus Moc. ex Ser.

Distribution: United States: California

\section{Diastrophus bassettii Beutenmüller, 1892}

Feeds on: Induces galls on Rubus procumbens Muhl. and R. hispidus L.

Distribution: United States: Connecticut, Massachusetts, North Carolina, New Jersey, New York, Rhode Island

\section{Diastrophus cuscutaeformis Osten Sacken, 1863}

Feeds on: Induces galls on Rubus L. spp.

Distribution: United States: Illinois, Indiana, Massachusetts, Maryland, Maine, Michigan, Minnesota, Missouri, North Carolina, New Hampshire, New Jersey, New York, Ohio, Pennsylvania, South Carolina, Tennessee, Virginia, Vermont; Canada: Newfoundland and Labrador, Ontario

\section{Diastrophus fragariae Beutenmüller, 1915}

Feeds on: Induces galls on Fragaria virginiana Duch.

Distribution: United States: Michigan, Minnesota; Canada: Ontario

\section{Diastrophus fusiformans Ashmead, 1890}

Feeds on: Induces galls on Potentilla L. sp.

Distribution: United States: Colorado, Washington 


\section{Diastrophus kincaidii Gillette, 1893}

Nomenclature:

Diastrophus minimus Bassett, 1900

Feeds on: Induces galls on Rubus nutkanus Moc. ex Ser. and R. parviflorus Nutt.

Distribution: United States: California, Idaho, Michigan, Minnesota, Montana, Oregon, Washington, Wisconsin; Canada: British Columbia, Ontario

\section{Diastrophus nebulosus (Osten Sacken, 1861)}

\section{Nomenclature:}

Cynips (Diastrophus?) nebulosus Osten Sacken, 1861

Feeds on: Induces galls on Rubus corchorifolius L. f. (=villosus)

Distribution: United States: Arkansas, Connecticut, District of Columbia, Delaware, Georgia, Illinois, Indiana, Kentucky, Louisiana, Massachusetts, Maryland, Michigan, Missouri, Mississippi, North Carolina, New Jersey, New York, Ohio, Oklahoma, Pennsylvania, South Carolina, Tennessee, Texas, Virginia, Vermont, West Virginia; Canada: Ontario

\section{Diastrophus niger Bassett, 1900}

Feeds on: Induces galls on Potentilla canadensis L.

Distribution: United States: Connecticut, Massachusetts, Minnesota, New Jersey, New York, Rhode Island

\section{Diastrophus piceus Provancher, 1886}

Feeds on: Gall unknown

Distribution: Canada: Ontario

\section{Diastrophus potentillae Bassett, 1864}

Nomenclature:

Gonaspis potentillae (Bassett, 1864)

Feeds on: Induces galls on Potentilla canadensis L. and P. simplex Michx.

Distribution: United States: Illinois, Indiana, Massachusetts, Maine, Minnesota, North Carolina, Ohio; Canada: New Brunswick, Nova Scotia 


\section{Diastrophus radicum Bassett, 1870}

Feeds on: Induces galls on Rubus corchorifolius L. f. (= villosus) and R. occidentalis L.

Distribution: United States: Colorado, Michigan, North Carolina

\section{Diastrophus smilacis Ashmead, 1896}

Feeds on: Host unknown; previously suspected to induce galls on Smilax rotundifolia L. and S. herbacea L., but this claim has been refuted (Gates et al. 2020)

Distribution: United States: Florida, Illinois

\section{Diastrophus tumefactus Kinsey, 1920}

Feeds on: Induces galls on Tridophyllum norvegicum (L.) Greene (= Potentilla monspeliensis var. norvegica)

Distribution: Canada: Ontario, Québec

Diastrophus turgidus Bassett, 1870

Nomenclature:

Diastrophus turdigus [sic] Bassett, 1870

Feeds on: Induces galls on Rubus strigosus Michx.

Distribution: Canada: Manitoba, Newfoundland and Labrador, Ontario, Québec; United States: Colorado, Maine, Minnesota, New York, Ohio, Vermont

\section{Genus Periclistus Förster, 1869}

\section{Periclistus arefactus McCracken \& Egbert, 1922}

Feeds on: Inquiline of: galls of Diplolepis arefacta (Gillette, 1894) on Rosa californica

Distribution: United States: California

\section{Periclistus californicus Ashmead, 1896}

Feeds on: Inquiline of: galls of Diplolepis polita (Ashmead, 1890)

Distribution: United States: California, Colorado, Wyoming 


\section{Periclistus obliquus Provancher, 1888}

Feeds on: Host unknown but presumed to be inquilinous

Distribution: United States: California

\section{Periclistus piceus Fullaway, 1911}

Feeds on: Inquiline of: galls of Diplolepis polita (Ashmead, 1890) on Rosa californica

Distribution: United States: California; Canada: Alberta, Ontario

\section{Periclistus pirata (Osten Sacken, 1863)}

Nomenclature:

Aulax pirata Osten Sacken, 1863 | Rhodites globulus Beutenmüller, 1892

Feeds on: Inquiline of: galls of Diplolepis ignota (Osten Sacken, 1863)

Distribution: United States: District of Columbia; Canada: Alberta, Ontario, Saskatchewan

Periclistus semipiceus (Harris, 1841)

Nomenclature:

Cynips semipiceus Harris, 1841

Feeds on: Inquiline of: undetermined subspherical galls on roots of Rosa sp.

Distribution: United States: Massachusetts

Periclistus smilacis Ashmead, 1896

Feeds on: Inquiline of: galls of Diastrophus smilacis Ashmead, 1896 [unverified]

Distribution: United States: Florida

Genus Synophromorpha Ashmead, 1903

Synophromorpha kaulbarsi Ritchie \& Shorthouse, 1987

Feeds on: Host unknown but presumed to be inquilinous

Distribution: Mexico: Puebla 


\section{Synophromorpha rubi Weld, 1952}

Feeds on: Inquiline of: galls of Diastrophus cuscutaeformis Osten Sacken, 1863 on Rubus spp.

Distribution: United States: Illinois, Massachusetts, Ohio, Rhode Island; Canada: Ontario

\section{Synophromorpha sylvestris (Osten Sacken, 1861)}

Nomenclature:

Synophrus sylvestris Osten Sacken, 1861

Feeds on: Inquiline of: galls of Diastrophus nebulosus (Osten Sacken, 1861); galls of Diastrophus turgidus Bassett, 1870; galls of Diastrophus bassettii Beutenmüller, 1892 on Rubus spp.

Distribution: United States: Arkansas, Connecticut, District of Columbia, Florida, Illinois, Indiana, Massachusetts, Maryland, Maine, Missouri, North Carolina, New Jersey, New York, Ohio, Oklahoma, Pennsylvania, Rhode Island, Tennessee, Virginia, West Virginia

\section{Synophromorpha terricola Weld, 1952}

Feeds on: Inquiline of: galls of Diastrophus bassettii Beutenmüller, 1892; galls of Diastrophus cuscutaeformis Osten Sacken, 1863; galls of Diastrophus radicum Bassett, 1870 on Rubus spp.

Distribution: United States: Alabama, Connecticut, Maryland, North Carolina, New York, Ohio, Virginia, West Virginia

\section{Tribe Diplolepidini}

\section{Genus Diplolepis Geoffroy, 1762}

Diplolepis arefacta (Gillette, 1894)

Nomenclature:

Rhodites arefactus Gillette, 1894

Feeds on: Induces galls on Rosa L. sp.

Distribution: United States: Colorado, Utah 


\section{Diplolepis ashmeadi (Beutenmüller, 1918)}

Nomenclature:

Rhodites ashmeadi Beutenmüller, 1918

Feeds on: Induces galls on Rosa nutkana C. Presl

Distribution: United States: Oregon

Diplolepis bassetti subsp. bassetti (Beutenmüller, 1918)

Nomenclature:

Rhodites bassetti Beutenmüller, 1918

Feeds on: Induces galls on Rosa nutkana C. Presl and R. woodsii Lindl.

Distribution: United States: Oregon; Canada: Alberta, British Columbia, Saskatchewan

Diplolepis bassetti subsp. lucida Kinsey, 1922

Nomenclature:

Diplolepis bassetti var. lucida Kinsey, 1922

Feeds on: Induces galls on Rosa nutkana C. Presl

Distribution: United States: Idaho, Oregon

Diplolepis bicolor (Harris, 1841)

Nomenclature:

Cynips bicolor Harris, 1841

Feeds on: Induces galls on Rosa woodsii Lindl. and R. arkansana Porter

Distribution: United States: Arkansas, California, Connecticut, Idaho, Illinois, Indiana, Kentucky, Maryland, Maine, Michigan, Minnesota, Missouri, Montana, North Carolina, New Hampshire, New Jersey, New York, Ohio, Pennsylvania, Washington, Wisconsin; Canada: Alberta, British Columbia, Manitoba, New Brunswick, Nova Scota, Ontario, Québec, Saskatchewan

\section{Diplolepis californica (Beutenmüller, 1914)}

Nomenclature:

Rhodites californicus Beutenmüller, 1914

Feeds on: Induces galls on Rosa L. sp. 
Distribution: United States: California, Idaho

Diplolepis dichlocera (Harris, 1841)

Nomenclature:

Cynips dichlocera Harris, 1841

Feeds on: Induces galls on Rosa L. sp.

Distribution: United States: Massachusetts; Canada: Ontario

Diplolepis fulgens (Gillette, 1894)

Nomenclature:

Rhodites fulgens Gillette, 1894

Feeds on: Induces galls on Rosa rugosa Thunb.

Distribution: United States: Illinois, New York, South Dakota

Diplolepis fusiformans subsp. fusiformans (Ashmead, 1890)

Nomenclature:

Rhodites fusiformans Ashmead, 1890

Feeds on: Induces galls on Rosa arkansana Porter, R. woodsii Lindl., and R. blanda Aiton

Distribution: United States: Arizona, Colorado, Idaho, Illinois, Minnesota, Nebraska; Canada: Alberta, British Columbia, Ontario

Diplolepis fusiformans subsp. mendocinensis Kinsey, 1922

Nomenclature:

Diplolepis fusiformans var. mendocinensis Kinsey, 1922

Feeds on: Induces galls on Rosa L. sp.

Distribution: United States: California

Diplolepis fusiformans subsp. minuta Kinsey, 1922

Nomenclature:

Diplolepis fusiformans var. minuta Kinsey, 1922

Feeds on: Induces galls on Rosa L. sp. 
Distribution: United States: California

Diplolepis gracilis (Ashmead, 1897)

Nomenclature:

Rhodites graxilis Ashmead, 1897

Feeds on: Induces galls on Rosa L. sp.

Distribution: United States: Minnesota, Wisconsin; Canada: Alberta, British Columbia, Saskatchewan

\section{Diplolepis ignota (Osten Sacken, 1863)}

\section{Nomenclature:}

Rhodites ignota Osten Sacken, 1863 | Rhodites carolina Ashmead, 1887

Feeds on: Induces galls on Rosa arkansana Porter, $R$. blanda Aiton, $R$. carolina L., $R$. nitida Willd., and $R$. virginiana Mill.

Distribution: United States: Colorado, Connecticut, District of Columbia, lowa, Illinois, Indiana, Massachusetts, Montana, North Carolina, New Jersey, New York, Pennsylvania, Washington; Canada: Manitoba, Saskatchewan

\section{Diplolepis inconspicuis Dailey \& Campbell, 1973}

Feeds on: Induces galls on Rosa californica Cham. and Schltdl.

Distribution: United States: California

\section{Diplolepis lens Weld, 1952}

Feeds on: Induces galls on Rosa nutkana C. Presl

Distribution: United States: California, Oregon, Washington

\section{Diplolepis mayri (Schlechtendal, 1877)}

\section{Nomenclature:}

Rhodites mayri Schlechtendal, 1877

Feeds on: Induces galls on Rosa rubiginosa L.

Distribution: United States: New Jersey 


\section{Diplolepis nebulosa (Bassett, 1890)}

Nomenclature:

Rhodites nebulosa Bassett, 1890

Feeds on: Induces galls on Rosa blanda Aiton, R. carolina L., R. rubiginosa L., and $R$. woodsii Lindl.

Distribution: United States: Connecticut, New York; Canada: Alberta, Ontario

\section{Diplolepis neglecta (Gillette, 1894)}

\section{Nomenclature:}

Rhodites neglecta Gillette, 1894

Feeds on: Induces galls on Rosa L. sp.

Distribution: United States: Colorado

\section{Diplolepis nervosa (Curtis, 1838)}

\section{Nomenclature:}

Diplolepis centifoliae (Hartig, 1840) | Rhodites centifoliae Hartig, $1840 \mid$ Rhodites andrei Kieffer, 1904 | Rhodites dispar Niblett, 1943 | Rhodites kiefferi Loiselle, 1912 | Diplolepis rosarum (Giraud, 1859) | Rhodites rosarum Giraud, 1859 | Cynips nervosa Curtis, 1838 |

Feeds on: Induces galls on Rosa canina L. and R. rubiginosa L.

Distribution: United States: Oregon; Canada: Ontario, Québec

\section{Diplolepis nodulosa (Beutenmüller, 1909)}

\section{Nomenclature:}

Rhodites nodulosus Beutenmüller, 1918

Feeds on: Induces galls on Rosa woodsii Lindl., Rosa virginiana Mill., and R. blanda Aiton; possibly induces galls onRosa carolina var. lucida (Ehrh.) Farw. [unsubstantiated]

Distribution: United States: Illinois, Massachusetts; Canada: Alberta, British Columbia, Ontario, Prince Edward Island, Saskatchewan 


\section{Diplolepis oregonensis (Beutenmüller, 1918)}

Nomenclature:

Rhodites oregonensis Beutenmüller, 1918

Feeds on: Induces galls on Rosa nutkana C. Presl

Distribution: United States: Idaho, Oregon, Washington; Canada: Saskatchewan

\section{Diplolepis ostensackeni (Beutenmüller, 1918)}

Nomenclature:

Rhodites ostensackeni Beutenmüller, 1918

Feeds on: Induces galls on Rosa nutkana C. PresI

Distribution: United States: Oregon

Diplolepis polita (Ashmead, 1890)

\section{Nomenclature:}

Rhodites polita Ashmead, 1890 | Rhodites occidentalis Beutenmüller, 1922

Feeds on: Induces galls on Rosa californica Cham. \& Schltdl., R. woodsii Lindl., $R$. acicularis Lindl., and $R$. arkansana Porter

Distribution: United States: Alaska, California, Colorado; Canada: Alberta, British Columbia, Manitoba, Ontario, Québec, Saskatchewan, Yukon

\section{Diplolepis pustulatoides (Beutenmüller, 1914)}

\section{Nomenclature:}

Rhodites pustulatoides Beutenmüller, 1914

Feeds on: Induces galls on Rosa L. sp.

Distribution: United States: Indiana

\section{Diplolepis radicum (Osten Sacken, 1863)}

\section{Nomenclature:}

Rhodites radicum Osten Sacken, 1863 | Tribalia batatorum Walsh, 1864 | Rhodites atahensis Bassett, 1890 | Diplolepis radicum var. johnsoni Kinsey, 1922 | Diplolepis radicum var. plana Kinsey, 1922 | Diplolepis radicum var. divergens Kinsey, 1922 | Diplolepis radicum var. angustior Hunter, 1923 
Feeds on: Produces galls on Rosa carolina L., R. nutkana C. Presl, and R. woodsii Lindl.

Distribution: United States: Colorado, Connecticut, District of Columbia, Illinois, Indiana, Massachusetts, Minnesota, North Carolina, New Jersey, New York, Ohio, Oregon, Pennsylvania, Washington; Canada: British Columbia, Manitoba, Ontario

\section{Diplolepis rosae (Linnaeus, 1758)}

Nomenclature:

Cynips rosae Linnaeus, 1758

Feeds on: Induces galls on Rosa rugosa Thunb., R. cinnamoea L., R. rubiginosa L., and $R$. blanda Aiton

Distribution: United States: California, Colorado, District of Columbia, Idaho, Illinois, Kansas, Massachusetts, Maine, Michigan, Montana, North Carolina, New Hampshire, New York, Ohio, Oregon, Pennsylvania, Rhode Island, South Carolina, Utah, Virginia, Vermont, Washington, West Virginia; Canada: Alberta, British Columbia, New Brunswick, Newfoundland and Labrador, Nova Scotia, Ontario, Québec

\section{Diplolepis rosaefolii (Cockerell, 1889)}

\section{Nomenclature:}

Rhodites rosaefolii Cockerell, 1889 | Rhodites lenticularis Bassett,

Feeds on: Induces galls on Rosa acicularis Lindl., R. woodsii Lindl., and R. arkansana Porter

Distribution: United States: California, Colorado, Illinois, Pennsylvania, Washington; Canada: Alberta, British Columbia, Manitoba, Newfoundland and Labrador, Nova Scotia, Ontario, Saskatchewan, Yukon

\section{Diplolepis similis (Ashmead, 1896)}

Nomenclature:

Rhodites similis Ashmead, 1897

Feeds on: Induces galls on Rosa L. sp.

Distribution: United States: Wyoming 


\section{Diplolepis spinosa (Ashmead, 1887)}

Nomenclature:

Rhodites spinosa Ashmead, 1887 | Rhodites spinosissima Gillette, 1889 | Rhodites multispinosa Gillette, 1890

Feeds on: Induces galls on Rosa rubiginosa L., R. woodsii Lindl., and R. blanda Aiton

Distribution: United States: California, Colorado, Florida, Idaho, Illinois, Massachusetts, Maine, Minnesota, Washington; Canada: Alberta, British Columbia, Manitoba, Ontario, Saskatchewan

\section{Diplolepis terrigena Weld, 1952}

Feeds on: Induces galls on Rosa L. sp.

Distribution: United States: California

\section{Diplolepis triforma Shorthouse \& Ritchie, 1984}

Feeds on: Induces galls on Rosa acicularis Lindl. and R. woodsii Lindl.

Distribution: United States: Minnesota; Canada: Alberta, Manitoba, Ontario, Saskatchewan

\section{Diplolepis tuberculator (Cockerell, 1888)}

\section{Nomenclature:}

Diplolepis tuberculatrix (Cockerell, 1888) | Rhodites tuberculator Cockerell, 1888 | Diplolepis tuberculatrix var. coloradensis form coloradensis Kinsey \& Ayres, 1922 | Diplolepis tuberculatrix var. coloradensis form subcoloradensis Kinsey \& Ayres, 1922 | Diplolepis tuberculatrix var. wasatchensis Kinsey \& Ayres, 1922 | Diplolepis tuberculatrix var. versicolor Kinsey \& Ayres, 1922 | Diplolepis tuberculatrix var. melanderi Kinsey \& Ayres, 1922 | Diplolepis tuberculatrix var. rubriderma Kinsey \& Ayres, 1922 | Diplolepis tuberculatrix var. sierranensis Kinsey \& Ayres, 1922 | Diplolepis tuberculatrix var. descansonis Kinsey \& Ayres, 1922

Feeds on: Induces galls on Rosa L. sp.

Distribution: United States: California, Colorado, North Dakota, New Mexico, Oregon, Utah, Washington

\section{Diplolepis tuberculosa (Osten Sacken, 1861)}

\section{Nomenclature:}

Cynips (Rhodites?) tuberculosa Osten Sacken, 1861 
Feeds on: Induces galls on Rosa L. sp.

Distribution: United States

Diplolepis tumida (Bassett, 1890)

Nomenclature:

Rhodites tumidus Bassett, 1890 | Diplolepis tuberculatrix var. tumida form xerophila Kinsey and Ayres, 1922

Feeds on: Produces galls on Rosa L. sp.

Distribution: United States: Utah

Diplolepis variabilis (Bassett, 1890)

Nomenclature:

Rhodites variabilis Bassett, 1890 | Rhodites globuloides Beutenmüller, 1907

Feeds on: Induces galls on Rosa woodsii Lindl.

Distribution: United States: Colorado, Itado, Texas, Utah, Washington, Wyoming; Canada: British Columbia

\section{Diplolepis verna (Osten Sacken, 1863)}

Nomenclature:

Rhodites verna Osten Sacken, 1863

Feeds on: Induces galls on Rosa blanda Aiton

Distribution: United States: District of Columbia, New York

\section{Diplolepis weldi (Beutenmüller, 1913)}

Nomenclature:

Rhodites weldi Beutenmüller, 1913

Feeds on: Induces galls on Rosa L. sp.

Distribution: United States: California 
Tribe Phanacidini

Genus Phanacis Förster, 1860

Phanacis hypochoeridis (Kieffer, 1887)

Nomenclature:

Aulax hypochaeridis Kieffer, 1887

Feeds on: Induces galls on Hypochaeris radicata L.

Distribution: United States: California, Oregon; Canada: British Columbia

Phanacis taraxaci (Ashmead, 1897)

Nomenclature:

Gillettea taraxaci Ashmead, 1897

Feeds on: Induces galls on Taraxacum officinale F. H. Wigg.

Distribution: United States: Minnesota, Pennsylvania

\section{Tribe Synergini}

Genus Saphonecrus Dalla Torre \& Kieffer, 1910

Saphonecrus favanus Weld, 1944

Distribution: United States: District of Columbia, Missouri

Saphonecrus gemmariae (Ashmead, 1885)

Distribution: United States: Florida

Genus Synergus Hartig, 1840

\section{Synergus agrifoliae Ashmead, 1896}

Nomenclature:

Synergus maculatus Fullaway, 1911 | Synergus obscurus McCracken \& Egbert, 1922

Feeds on: Inquiline of: galls of Neuroterus saltatorius (Edwards, 1874); undetermined round, brown galls, $2.0 \mathrm{~mm}$ in diameter located on adaxial leaf surface on Quercus agrifolia (Lobato-Vila and Pujade-Villar 2021); undetermined galls on Quercus garryana Douglas ex Hook 
Distribution: United States: California

Synergus ashmeadi Lobato-Vila \& Pujade-Villar, 2021

Feeds on: Inquiline of: galls of Andricus sp. on Quercus potosina

Distribution: Mexico: Aguascalientes

Synergus aurofacies Lobato-Vila \& Pujade-Villar, 2020

Feeds on: Inquiline of: galls of Femuros Iusum Kinsey, 1937

Distribution: Mexico: Ciudad de México

Synergus atra Gillette, 1896

Feeds on: Inquiline of: galls of Disholcaspis rubens (Gillette, 1893) (asexual generation)

Distribution: United States: Colorado, Michigan

Synergus atripennis Ashmead, 1896

Feeds on: Inquiline of: galls of Disholcaspis spongiosa (Karsch, 1880) (asexual generation)

Distribution: United States: Florida

Synergus atripes Gillette, 1896

Feeds on: Inquiline of: galls of Atrusca brevipennata (Gillette, 1893) (asexual generation)

Distribution: United States: Colorado

Synergus batatoides Ashmead, 1885

Feeds on: Inquiline of: galls of Callirhytis quercusbatoides (Ashmead, 1881) (asexual generation)

Distribution: United States: Florida

Synergus bellus McCracken \& Egbert, 1922

Feeds on: Inquiline of: undetermined galls on Quercus chrysolepis Liebm.

Distribution: United States: California 


\section{Synergus beutenmulleri Lobato-Vila \& Pujade-Villar, 2021}

Feeds on: Inquiline of: galls of Andricus sp. Q. potosina

Distribution: Mexico: Aguascalientes

\section{Synergus brevicornis Ashmead, 1896}

Nomenclature:

Saphonecrus brevicornis (Ashmead, 1896)

Feeds on: Inquiline of: galls of Andricus sp. on Quercus wislizeni A. DC.

Distribution: United States: California

\section{Synergus bicolor Ashmead, 1885}

Feeds on: Inquiline of: galls of Andricus quercusfoliatus (Ashmead, 1881)

Distribution: United States: Florida

\section{Synergus campanula Osten Sacken, 1865}

Feeds on: Inquiline of: galls of Andricus dimorphus (Beutenmüller, 1913); galls of Acraspis erinacei (Beutenmüller, 1909); galls of Acraspis pezomachoides (Osten Sacken, 1862); galls of Disholcaspis quercusglobulus (Fitch, 1859); galls of Disholcaspis quercusmamma (Walsh, 1869); galls of Trigonaspis ? quercusforticorne (Walsh, 1864)

Distribution: United States: District of Columbia, Illinois, lowa, Kansas, Kentucky, Missouri, New Jersey, New York, Wisconsin

\section{Synergus castanopsidis (Beutenmüller, 1918)}

Nomenclature:

Periclistus castanopsidis (Beutenmüller, 1918)

Feeds on: Inquiline of: galls of Dryocosmus castanopsidis (Beutenmüller, 1917) (asexual generation)

Distribution: United States: California

\section{Synergus cibriani Pujade-Villar \& Lobato-Vila, 2017}

Feeds on: Inquiline of: undetermined spherical galls, possibly induced by Disholcaspis, on twigs of undetermined species of Quercus; undetermined spherical galls on abaxial leaf surface on Quercus glabrescens (Lobato-Vila and Pujade-Villar 2021) 
Distribution: Mexico: Michoacán de Ocampo

\section{Synergus citriformis (Ashmead, 1885)}

Nomenclature:

Ceroptres citriformis Ashmead, 1885 | Synergus niger Fullaway, 1911 | Synergus elegans Nieves-Aldrey \& Medianero, 2011

Feeds on: Inquiline of: "... a gall related to Amphibolips citriformis Ashmead" (LobatoVila and Pujade-Villar 2021); galls of Amphibolips, Disholcaspis, Cynips, Andricus, Dros, and Antron spp. on Quercus spp. in sections Quercus and Lobatae (Lobato-Vila and Pujade-Villar 2021).

Distribution: United States: California, Florida; Mexico: Aguascalientes, Ciudad de México, Guanajuato, Hidalgo, México, Michoacán de Ocampo, Morelos, Oaxaca, Puebla, Tlaxcala, Veracruz de Ignacio de la Llave, Zacatecas

\section{Synergus compressus Lobato-Vila \& Pujade-Villar, 2021}

Feeds on: Inquiline of: tuberous galls probably induced by Andricus on Quercus crassifolia

Distribution: Mexico: Puebla

\section{Synergus confertus McCracken \& Egbert, 1922}

Feeds on: Inquiline of: galls of Andricus confertus McCracken \& Egbert, 1922

Distribution: United States: California

\section{Synergus coniferae Ashmead, 1885}

Feeds on: Inquiline of: galls of Callirhytis quercusventricosa (Bassett, 1864)

Distribution: United States: Florida, lowa, Missouri

\section{Synergus digressus McCracken \& Egbert, 1922}

Feeds on: Inquiline of: undetermined twig galls on Quercus agrifolia Née

Distribution: United States: California

\section{Synergus dimorphus Osten Sacken, 1865}

Feeds on: Inquiline of: "woody twig galls on red oaks" (Gillette 1896); galls of Besbicus conspicuus (Kinsey, 1930) on Quercus lobata (Lobato-Vila and Pujade-Villar 2021) 
Distribution: United States: California, District of Columbia, Michigan

\section{Synergus distinctus McCracken \& Egbert, 1922}

Feeds on: Inquiline of: galls of Disholcaspis canescens (Bassett, 1890)

Distribution: United States: California

\section{Synergus diversicolor Lobato-Vila \& Pujade-Villar, 2021}

Feeds on: Inquiline of: galls of Melikaiella bicolor Pujade-Villar, 2014; galls of Sphaeroteras sp. on the leaves of Quercus obtusata; galls of Neuroterus sp. on the leaves of Quercus peduncularis; undetermined round galls on the twigs of Quercus acutifolia

Distribution: Mexico: Hidalgo, Jalisco, Morelos, Puebla

\section{Synergus dorsalis (Provancher, 1888)}

\section{Nomenclature:}

Synergus splendidus Fullaway, 1911 | Synergus dubiosus Fullaway, 1911 | Ceroptres dorsalis Provancher, 1888

Feeds on: Inquiline of: undetermined galls on Quercus lobata; galls of Amphibolips quercuspomiformis (Bassett 1881) on Quercus agrifolia; Synergus dubiosus was reared from galls of $A$. quercuspomiformis on $Q$. agrifolia; undetermined galls on Quercus wislizeni. See Lobato-Vila and Pujade-Villar (2021) for more details.

Distribution: United States: California

\section{Synergus duricorius Gillette, 1896}

Feeds on: Inquiline of: galls of Disholcaspis quercusmamma (Walsh, 1869)

Distribution: United States: Delaware, Minnesota

\section{Synergus ebenus Lobato-Vila \& Pujade-Villar, 2021}

Feeds on: Inquiline of: galls of Femuros repandae Kinsey, 1937; undetermined galls of Neuroterus on the leaves of Quercus obtusata; undetermined galls of Andricus on the twigs of Quercus obtusata

Distribution: Mexico: Ciudad de México, México, Veracruz de Ignacio de la Llave

\section{Synergus equihuai Pujade-Villar \& Lobato-Vila, 2016}

Feeds on: Inquiline of: undetermined concealed galls in the acorns of Quercus rugosa 
Distribution: Mexico: Morelos

\section{Synergus erinacei Gillette, 1896}

Feeds on: Inquiline of: galls of Acraspis erinacei (Beutenmüller, 1909); galls of Acraspis macrocarpae Bassett, 1890; galls of Acraspis pezomachoides Osten Sacken, 1862

Distribution: United States: lowa, Illinois, Kentucky, New York; Canada: Ontario

\section{Synergus estradae Pujade-Villar \& Lobato-Vila, 2016}

Feeds on: Inquiline of: woody, tuberous galls induced by Andricus spp. (Lobato-Vila and Pujade-Villar 2021)

Distribution: Mexico: Hidalgo, Morelos, Puebla

\section{Synergus ficigerae Ashmead, 1885}

Feeds on: Inquiline of: galls of Disholcaspis sp. on Quercus magnoliifolia

Distribution: United States: Florida, Virginia

\section{Synergus filicornis Cameron, 1883}

\section{Nomenclature:}

Synergus similis Gillette, 1896 | Synergus furnessana Weld, 1913

Feeds on: Inquiline of: woolly galls of Andricus furnessae (Weld, 1913); woolly galls of Andricus mexicanus Kinsey (= Andricus nievesaldreyi Pujade-Villar, 2011); woolly galls of Andricus crystallinus; woolly galls of Andricus georgei; woolly galls of Andricus maesi; woolly galls of Cynips spp. (Antron group); woolly galls of Andricus nievesaldreyi; woolly galls of Andricus quercuslanigera (Ashmead) (= A. linaria (Kinsey)); non-woolly galls of Andricus rochai Pujade-Villar, 2018; non-woolly galls of Atrusca spp.; non-woolly galls of Disholcaspis nr. insulana; non-woolly galls of Disholcaspis nr. mexicana; non-woolly galls of Kokkocynips nr. doctorrosae; non-woolly galls of Loxaulus; undetermined galls on Quercus sect. Quercus. According to LobatoVila and Pujade-Villar (2021), Synergus filicornis was described from specimens "obtained from woolly leaf galls of 'Andricus guatemalensis (Cameron, 1883)'", a name they consider incertae sedis.

Distribution: Mexico: Aguascalientes, Ciudad de México, Guanajuato, Jalisco, México, Michoacán de Ocampo, Morelos, Puebla, Querétaro, Zacatecas 


\section{Synergus flavens McCracken \& Egbert, 1922}

Nomenclature:

Synergus variegatus McCracken \& Egbert, 1922

Feeds on: Inquiline of: galls of Heteroecus flavens (McCracken \& Egbert, 1922); galls of Heteroecus pacificus (Ashmead, 1896); galls of Trichoteras coquilletti Ashmead, 1897

Distribution: United States: California

\section{Synergus forcadellae Lobato-Vila \& Pujade-Villar, 2020}

Feeds on: Inquiline of: woolly galls of Andricus $\mathrm{sp}(\mathrm{p})$.; woolly galls of Striatoandricus $\mathrm{sp}(\mathrm{p})$.; non-woolly galls of Andricus spp.; non-woolly galls of Atrusca spp.; non-woolly galls of Amphibolips spp.; non-woolly galls of Cynips spp. mainly on Quercus sect. Quercus (Lobato-Vila and Pujade-Villar 2021)

Distribution: Mexico: Ciudad de México, Guanajuato, México, Michoacán de Ocampo, Morelos, Querétaro

\section{Synergus gilletti Pujade-Villar \& Lobato-Vila, 2017}

Feeds on: Inquiline of: galls of Atrusca sp. on Quercus laeta and Quercus rugosa; galls of Andricus on the leaves of Quercus laeta

Distribution: Mexico: Zacatecas

\section{Synergus grahami Lobato-Vila \& Pujade-Villar, 2019}

Feeds on: Inquiline of: galls of Andricus santafe Pujade-Villar, 2013; undetermined galls, possibly Andricus or Loxaulus, on Quercus crassipes and Quercus laeta; galls of Loxaulus laeta Pujade-Villar, 2014

Distribution: Mexico: Ciudad de México

\section{Synergus incisus Gillette, 1896}

Feeds on: Inquiline of: galls of Callirhytis frequens (Gillette, 1892)

Distribution: United States: Colorado, New Mexico

\section{Synergus laeviventris (Osten Sacken, 1861)}

Feeds on: Inquiline of: galls of Amphibolips cookii Gillette, 1888; galls of Amphibolips confluenta (Harris, 1841); galls of Amphibolips quercusinanis Osten Sacken, 1862; galls of Amphibolips quercusjuglans (Osten Sacken, 1862); galls of Amphibolips 
quercusostensackenii (Bassett, 1863); galls of Amphibolips quercusspongifica (OstenSacken, 1862); galls of Andricus pisiformis Beutenmüller, 1911; galls of Andricus quercusfrondosus (Bassett, 1865); galls of Atrusca quercuscentricola (Osten-Sacken, 1861); galls of Disholcaspis quercusglobulus (Fitch, 1839); galls of Disholcaspis rubens (Gillette, 1893); undetermined gall on Quercus falcata Michx.; undetermined bud gall on undetermined Quercus sect. Lobatae

Distribution: United States: Colorado, District of Columbia, Illinois, Iowa, Michigan, Missouri, Ohio, Pennsylvania

\section{Synergus lignicola (Osten Sacken, 1862)}

\section{Nomenclature:}

Cynips (Synerges [sic]) lignicola Osten Sacken, 1862 | Synergus davisi (Beutenmüller, 1907) | Andricus davisi Beutenmüller, 1907 | Synerges [sic] rhoditiformis Walsh, 1864

Feeds on: Inquiline of: galls of Andricus quercuslaurinus Melika \& Pujade-Villar, 2009; galls of Callirhytis cornigera (Osten-Sacken, 1865); galls of Callirhytis quercusgemmaria (Ashmead, 1885); galls of Callirhytis quercuspunctata (Bassett, 1863); undetermined galls on Quercus incana and other Quercus sect. Lobatae spp.

Distribution: United States: District of Columbia, Indiana, Michigan, Missouri, New Jersey, New York, Virginia

\section{Synergus linnei Lobato-Vila \& Pujade-Villar, 2021}

Feeds on: Inquiline of: small, round galls of Disholcaspis sp. on twigs of Quercus sp.; small, striped, round, undetermined galls on twigs of Quercus sp. (see Lobato-Vila and Pujade-Villar 2021)

Distribution: Mexico: Querétaro, Zacatecas

\section{Synergus longimalaris Pujade-Villar \& Lobato-Vila, 2017}

Feeds on: Inquiline of: galls of Amphibolips dampfi Kinsey, 1937; galls of Amphibolips sp. on Quercus affinis Scheidw., Q. conzattii Trel., and Q. glabrescens Benth.; galls of Amphibolips sp. on Quercus sp.; galls of Andricus tecturnarum species group on Quercus sp. (see Lobato-Vila and Pujade-Villar 2021)

Distribution: Mexico: Hidalgo, Jalisco, México, Michoacán de Ocampo, Morelos

\section{Synergus longiscapus Pujade-Villar \& Lobato-Vila, 2017}

Feeds on: Inquiline of: galls of Disholcaspis sp. on Quercus candicans Née, Q. eduardi c.f., Q. emoryi Torr., Q. glabrescens Benth., Q. laeta, and Q. rugosa; galls of Andricus sp. on Quercus candicans Née, Q. eduardi c.f., Q. emoryi Torr., Q. glabrescens Benth., 
Q. laeta, and Q. rugosa; unidentified subellipsoidal galls on the leaves of on Quercus candicans Née, Q. eduardi c.f., Q. emoryi Torr., Q. glabrescens Benth., Q. laeta, and Q. rugosa; galls of Neuroterus eugeros Pujade-Villar; galls of the Andricus georgei and Andricus maesi groups; galls of Andricus sphaericus Pujade-Villar; galls of undetermined species of Andricus, Cynips, and Disholcaspis on Quercus sect. Quercus spp. (see Lobato-Vila and Pujade-Villar 2021)

Distribution: Mexico: Ciudad de México, México, Morelos, Nuevo León, Oaxaca, Tlaxcala, Veracruz de Ignacio de la Llave, Zacatecas

\section{Synergus macrackenae Lobato-Vila \& Pujade-Villar, 2021}

\section{Nomenclature:}

Synergus nigro-ornatus McCracken \& Egbert, 1922

Feeds on: Inquiline of: galls of Loxaulus hyalinus Pujade-Villar \& Melika, 2014; galls of Andricus fusiformis Pujade-Villar, 2014; galls of Neuroterus sp. on leaves of Quercus obtusata

Distribution: Mexico: México, Michoacán de Ocampo

\section{Synergus medullae Ashmead, 1885}

Feeds on: Inquiline of: galls of Zapatella quercusmedullae (Ashmead, 1885) on Quercus incana Bartram (= Q. cinerea Raf.), Q. marilandica (L.) Münchn. and $Q$. myrtifolia Willd.

Distribution: United States: Florida

\section{Synergus mendax Walsh, 1864}

\section{Nomenclature:}

Synerges [sic] mendax Walsh, 1864

Feeds on: Inquiline of: galls of Callirhytis quercuspunctata (Bassett, 1863)

Distribution: United States: Illinois

\section{Synergus mexicanus Gillette, 1896}

\section{Nomenclature:}

Synophrus mexicanus (Gillette, 1896) | Synergus mexicana Gillette, 1896 | Synergus dugesi Ashmead, 1899 | Synergus multiplicatus Fullaway, 1911 | Saphonerus brevis Weld, 1926 | Synergus brevis (Weld, 1926) 
Feeds on: Inquiline of: "woody tuberous galls and fusiform twig swellings ... of oaks from both Quercus and Lobatae sections" (Lobato-Vila and Pujade-Villar 2021); galls of Andricus chrysolepidicola (Ashmead, 1896) (= Andricus kelloggi (Fullaway, 1911)) on Quercus douglasii Hook. and Arn. (Quercus section) (see Lobato-Vila and Pujade-Villar 2021).

Distribution: United States: Arizona, California, New Mexico; Mexico: Ciudad de México, Guanajuato, Hidalgo, México, Zacatecas

\section{Synergus nigroornatus McCracken \& Egbert, 1922}

Feeds on: Inquiline of: galls of Heteroecus pacificus (Ashmead, 1896)

Distribution: United States: California

\section{Synergus oaxaquensis Lobato-Vila \& Pujade-Villar, 2021}

Feeds on: Inquiline of: galls of an undetermined genus, possibly Andricus or Loxaulus, on twigs of Quercus obtusata (see Lobato-Vila and Pujade-Villar 2021)

Distribution: Mexico: Oaxaca

\section{Synergus obtusilobae (Ashmead, 1885)}

Nomenclature:

Ceroptres obtusilobae Ashmead, 1885

Feeds on: Inquiline of: undetermined gall on Quercus stellata; undetermined gall on Quercus alba L. (see Lobato-Vila and Pujade-Villar 2021)

Distribution: United States: District of Columbia, Florida, Missouri, Virginia

\section{Synergus ochreus Fullaway, 1911}

Feeds on: Inquiline of: galls of Besbicus conspicuus (Kinsey, 1930) (= Cynips ( Besbicus) multipunctata var. conspicua) on Quercus lobata; galls of Amphibolips quercuspomiformis (Bassett, 1881); galls of Disholcaspis eldoradensis (Beutenmüller, 1909); galls of Disholcaspis sp. on Quercus oblongifolia; undetermined galls on Quercus lobata and Q. dumosa (see Lobato-Vila and Pujade-Villar 2021)

Distribution: United States: Arizona, California 


\section{Synergus oneratus (Harris, 1841)}

\section{Nomenclature:}

Cynips oneratus Harris, 1841 | Synergus garryanus Gillette, 1893 | Synergus oneratus coloradensis (Gillette, 1896) | Synergus oneratus var. coloradensis Gillette, 1896

Feeds on: Inquiline of: galls of Andricus (=Adleria) quercusstrobilana (Osten-Sacken, 1862); galls of Atrusca brevipennata (Gillette, 1893); galls of Disholcaspis quercusglobulus (Fitch, 1859); galls of Disholcaspis rubens (Gillette, 1893); galls similar to those of Disholcaspis perniciosa (Bassett, 1890) (= Holcaspis monticola Gillette, 1893) on Quercus garryana; galls of Disholcaspis eldoradensis on Quercus lobata and Q. dumosa; galls of Disholcaspis perniciosa on Quercus gambelii; galls of Disholcaspis rubens on Quercus x undulata; galls of Acraspis erinacei (Beutenmüller, 1909); galls of Acraspis pezomachoides Osten Sacken, 1862; galls of Philonix nigra Gillette, 1889; galls of Atrusca quercuscentricola (Osten Sacken, 1861); galls of Andricus robustus Weld, 1926; galls of Andricus biconicus Weld, 1926; galls of Disholcaspis quercusmamma (Walsh, 1869) (see Lobato-Vila and Pujade-Villar (2021) and references therein)

Distribution: United States: California, Colorado, District of Columbia, lowa, Illinois, Kentucky, Massachusetts, Michigan, Missouri, New Jersey, New York, Ohio, Oregon, Tennessee, Washington; Canada: Ontario

\section{Synergus pacificus McCracken \& Egbert, 1922}

\section{Nomenclature:}

Synergus profusus McCracken \& Egbert, 1922

Feeds on: Inquiline of: galls of Heteroecus pacificus (Ashmead, 1896) (= Andricus pacificus) on Quercus chrysolepis; galls of Disholcaspis canescens (Bassett, 1890) on Quercus douglasii (see Lobato-Vila and Pujade-Villar 2021 and references therein)

Distribution: United States: California

\section{Synergus personatus Lobato-Vila \& Pujade-Villar, 2021}

Feeds on: Inquiline of undetermined species of Atrusca on $Q$. microphylla and $Q$. glabrescens

Distribution: Mexico: México, Tlaxcala

\section{Synergus pomiformis Ashmead, 1885}

\section{Nomenclature:}

Ceroptres pomiformis Ashmead, 1885 | Synergus flavus Kieffer, 1904 | Synergus varicolor Fullaway, 1911 | Synergus variegatus McCracken \& Egbert, 1922 
Feeds on: Inquiline of: galls of Callirhytis quercuspomiformis (Bassett, 1881) (= C. maculipennis Kieffer, 1904) on Quercus wislizeni A. DC. and Q. agrifolia (Lobatae section); Amphibolips sp. on Quercus agrifolia (Lobato-Vila and Pujade-Villar 2021)

Distribution: United States: California; Mexico: Baja California

\section{Synergus pseudofilicornis Lobato-Vila \& Pujade-Villar, 2018}

Feeds on: Inquiline of: galls of Andricus quercuslaurinus Melika \& Pujade-Villar, 2009 (asexual generation) on Quercus affinis and Quercus laurina; galls of Andricus quercuslaurinus (sexual generation); other tuberous galls initiated by Andricus spp.; woolly galls induced by Andricus spp. and Striatoandricus spp.; non-woolly galls induced by Andricus, Atrusca, Cynips, Dros, Neuroterus on Quercus spp. (sections Quercus and Lobatae) (Lobato-Vila and Pujade-Villar 2021)

Distribution: Mexico: Ciudad de México, Hidalgo, México, Morelos, Nuevo León, Puebla, Tlaxcala, Veracruz de Ignacio de la Llave

\section{Synergus punctatus Gillette, 1896}

\section{Nomenclature:}

Synergus punctata Gillette, 1896

Feeds on: Inquiline of: galls of Disholcaspis rubens (Gillette, 1893) and Disholcaspis eldoradensis (Beutenmüller, 1909) and Biorhiza eburnea (Bassett, 1890) (= Dryophanta glabra Gillette, 1894) on Quercus arizonica Sarg., Q. dumosa Nutt. (= Q. durata), Q. gambelii Nutt., Q. garryana Dougl. ex Hook., Q. lobata Née, Q. oblongifolia Torr., Q. rugosa, Q. toumeyi Sarg., Q. turbinella Greene and Q. undulata Torr.; galls of Andricus nigricens (Gillette, 1888); galls of Philonix nigra (Gillette, 1889); galls of Acraspis erinacei (Beutenmüller, 1909); galls of Acraspis macrocarpae Bassett, 1890; galls of Andricus robustus Weld, 1926 (Lobato-Vila and Pujade-Villar 2021)

Distribution: United States: California, Colorado, Illinois, lowa, Kansas, Kentucky, Missouri

\section{Synergus quercuslana (Fitch, 1859)}

\section{Nomenclature:}

Cynips quercus-lana Fitch, 1859 | Andricus lana Ashmead, 1885 | Synergus lana Cresson, 1887

Feeds on: Inquiline of Andricus quercusflocci (Walsh, 1864)

Distribution: United States: lowa, New York 


\section{Synergus reniformis McCracken \& Egbert, 1922}

Nomenclature:

Synergus magnificus Weld, 1957

Feeds on: Inquiline of: galls of Disholcaspis ? reniformis (McCracken \& Egbert, 1922) on Quercus vaccinifolia; galls of Andricus ? truckeensis (Ashmead, 1896) on Quercus chrysolepis; unknown galls on Quercus chrysolepis and Quercus vaccinifolia (LobatoVila and Pujade-Villar 2021)

Distribution: United States: California, Oregon; Mexico: Baja California

\section{Synergus ruficephalus Lobato-Vila \& Pujade-Villar, 2021}

Feeds on: Inquiline of: galls induced by Andricus, possibly A. quercuslaurinus Melika \& Pujade-Villar, 2009, on Quercus crassipes (Lobato-Vila and Pujade-Villar 2021)

Distribution: Mexico: México

\section{Synergus rutulus McCracken \& Egbert, 1922}

Feeds on: Inquiline of Disholcaspis plumbella Kinsey, 1920

Distribution: United States: California

\section{Synergus shorthousei Lobato-Vila \& Pujade-Villar, 2019}

Feeds on: Inquiline of: "woody tuberous galls of different Andricus species on twigs of oaks of both Quercus and Lobatae sections" (Lobato-Vila and Pujade-Villar 2021)

Distribution: Mexico: Guanajuato, Hidalgo, México, Michoacán de Ocampo, Oaxaca, Zacatecas

\section{Synergus stelluli Burnett, 1976}

Feeds on: Inquiline of: galls of Andricus stellulus Burnett, 1974

Distribution: United States: California

\section{Synergus striatifrons Pujade-Villar \& Lobato-Vila, 2017}

Feeds on: Inquiline of: galls of Amphibolips hidalgoensis Pujade-Villar \& Melika, 2011 (asexual generation) on Quercus eduardi and Q. crassipes; galls of Amphibolips zacatequensis Melika \& Pujade-Villar, 2011 on Quercus eduardi and Q. crassipes; galls of Amphibolips cibriani Pujade-Villar, 2018 (asexual generation) on Quercus crassipes (see Lobato-Vila and Pujade-Villar 2021) 
Distribution: Mexico: Ciudad de México, Hidalgo, México, Tlaxcala, Zacatecas

\section{Synergus succinipedis (Ashmead, 1885)}

Nomenclature:

Ceroptres succinipedis Ashmead, 1885

Feeds on: Inquiline of: galls of Disholcaspis quercusvirens (Ashmead, 1881)

Distribution: United States: Florida

\section{Synergus tenebrosus Lobato-Vila \& Pujade-Villar, 2019}

Feeds on: Inquiline of: woody tuberous twig galls of Andricus sp. on Quercus mexicana Bonpl. (Lobatae Section) and Q. rugosa (Quercus section)

Distribution: Mexico: Guerrero, México

\section{Synergus villosus Gillette, 1891}

Feeds on: Inquiline of: galls of Acraspis villosa Gillette, 1888, Callirhytis lanata (Gillette, 1891), and Dryocosmus imbricariae (Ashmead, 1896)

Distribution: United States: Illinois, lowa

\section{Synergus virentis (Ashmead, 1885)}

\section{Nomenclature:}

Ceroptres virentis Ashmead, 1885

Feeds on: Inquiline of: galls of Disholcaspis quercusvirens (Ashmead, 1881)

Distribution: United States: Florida

\section{Synergus walshii Gillette, 1896}

\section{Nomenclature:}

Synophrus albipes Walsh, 1864 | Synergus albipes (Walsh, 1864)

Feeds on: Inquiline of: galls of Andricus ignotus (Bassett, 1900), Andricus pattoni (Bassett, 1881), Andricus quercusflocci (Walsh, 1864), Phylloteras volutellae Ashmead, 1897, and Phylloteras poculum (Osten Sacken, 1862)

Distribution: United States: Illinois, lowa, Kentucky, Michigan, Missouri 


\section{Synergus weldi Lobato-Vila \& Pujade-Villar, 2021}

Feeds on: Inquiline of: galls of Andricus sp. on twigs of Quercus mexicana (Lobato-Vila and Pujade-Villar 2021)

Distribution: Mexico: Guanajuato

\section{Discussion}

Examination of cynipids from the covered geographic range yields 170 valid species amongst 323 published taxonomic names (see Table 1).

Table 1.

Table 1: Species diversity by country

\begin{tabular}{|c|c|c|c|c|c|}
\hline Tribe & Genus & Total Species & United States & Canada & Mexico \\
\hline \multirow[t]{4}{*}{ Aulacideini } & & 21 & 20 & 6 & 0 \\
\hline & Antistrophus & 10 & 10 & 1 & 0 \\
\hline & Aulacidea & 10 & 9 & 4 & 0 \\
\hline & Liposthenes & 1 & 1 & 1 & 0 \\
\hline \multirow[t]{3}{*}{ Ceroptresini } & & 19 & 13 & 1 & 7 \\
\hline & Buffingtonella & 1 & 1 & 0 & 0 \\
\hline & Ceroptres & 18 & 12 & 1 & 7 \\
\hline \multirow[t]{4}{*}{ Diastrophini } & & 25 & 20 & 11 & 1 \\
\hline & Diastrophus & 14 & 12 & 8 & 0 \\
\hline & Periclistus & 7 & 5 & 2 & 0 \\
\hline & Synophromorpha & 4 & 3 & 1 & 1 \\
\hline Diplolepidini & Diplolepis & 34 & 34 & 16 & 0 \\
\hline Phanacidini & Phanacis & 2 & 2 & 1 & 0 \\
\hline \multirow[t]{3}{*}{ Synergini } & & 69 & 44 & 2 & 29 \\
\hline & Saphonecrus & 2 & 2 & 0 & 0 \\
\hline & Synergus & 67 & 42 & 2 & 29 \\
\hline Total species & & 170 (Total) & 133 (USA) & 38 (CAN) & 37 (MEX) \\
\hline
\end{tabular}

Kinsey and Ayres (1922) apparently amended the original spelling of Ashmead's Diplolepis tuberculator as Diplolepis tuberculatrix without explanation. The amended name was subsequently carried forward in publications by other authors, particularly in Burks (1979). We treat Kinsey and Ayres's spelling as an unjustified emendation, as described in Article 
33.2.3 of the International Code of Zoological Nomeclature (Fourth Edition) and revert to the spelling to Ashmead's original Diplolepis tuberculator.

Our experience in developing this catalogue reveals the need for a better mechanism to record and share data on extended phenotypes and for fine-grained trait data more generally. A model has been developed for the semantic representation of phenotype data (Balhoff et al. 2013, Mikó et al. 2014), for example, and further development of the Taxon Description extension or even a new phenotype-focused Darwin Core extension might be a convenient vehicle for sharing these data.

\section{Acknowledgements}

We are exceptionally grateful to Y. Miles Zhang for personal commentary on the taxonomy and distribution of Diplolepidini, John F. Tooker for personal commentary on the taxonomy, distribution, and natural history of Aulacideini, to Norm Johnson for the use of data from the Hymenoptera Online database, and to Johan Liljeblad for his essential contributions to cynipid data in the Hymenoptera Online database. Katja Seltmann and Jorrit Poelen provided much needed feedback regarding the recording of association data. Matt Yoder, Y. Miles Zhang, and an anonymous reviewer provided incredibly useful feedback on the final version of this manuscript.

\section{References}

- Balhoff JP, Mikó I, Yoder MJ, Mullins PL, Deans AR (2013) A semantic model for species description applied to the ensign wasps (Hymenoptera: Evaniidae) of New Caledonia. Systematic Biology 62 (5): 639-659. https://doi.org/10.1093/sysbio/syt028

- Buffington ML, Forshage M, Liljeblad J, Tang C, van Noort S (2020) World Cynipoidea (Hymenoptera): A key to higher-level groups. Insect Systematics and Diversity 4 (4). https://doi.org/10.1093/isd/ixaa003

- $\quad$ Burks BD (1979) Superfamily Cynipoidea. In: Krombein KV, Hurd P, Smith D, Burks BD (Eds) Catalog of Hymenoptera in America north of Mexico. 1. Smithsonian Institution Press, Washington, D.C., 1198 pp. URL: https://archive.org/details/ catalogofhymenop01 krom

- Deans AR, Nastasi LF, Montelongo DC (2021) Glossary of gall terms. 2. The Pennsylvania State University. Release date: 2021-3-02. URL: http://doi.org/ 10.26207/22e9-ck06

- $\quad$ Gates M, Zhang Y, Buffington M (2020) The great greenbriers gall mystery resolved? New species of Aprostocetus Westwood (Hymenoptera, Eulophidae) gall inducer and two new parasitoids (Hymenoptera, Eurytomidae) associated with Smilax L. in southern Florida, USA. Journal of Hymenoptera Research 80 (71). https://doi.org/10.3897/jhr. 80.59466

- Gillette CP (1896) A monograph of the genus Synergus Hartig. Transactions of the American Entomological Society 23 (1): 85-100. URL: http://www.jstor.org/stable/ $\underline{25076660}$

- $\quad$ iNaturalist (2021) iNaturalist. https://www.inaturalist.org. Accessed on: 2021-3-08. 
- Johnson N, Buffington M, Liljeblad J (2019) Cynipoidea (gall wasps) - xBio:D Cyberinfrastructure, The Ohio State University. 73.38. Museum of Biological Diversity, The Ohio State University. Release date: 2018-8-29. URL: https://doi.org/10.15468/ mdawzp

- $\quad$ Kinsey A, Ayres K (1922) Studies of some new and described Cynipidae (Hymenoptera), Indiana University Studies no. 53. University of Indiana, Bloomington.

- Lobato-Vila I, Pujade-Villar J (2021) The genus Synergus Hartig (Hymenoptera: Cynipidae: Synergini) in the New World: a complete taxonomic revision with a key to species. Zootaxa 4906 (1): 1-121. https://doi.org/10.11646/zootaxa.4906.1.1

- Mikó I, Copeland R, Balhoff J, Yoder M, Deans A (2014) Folding wings like a cockroach: a review of transverse wing folding ensign wasps (Hymenoptera: Evaniidae: Afrevania and Trissevania. PLoS One 9 (5): e94056. https://doi.org/10.1371/journal.pone.0094056

- Poelen J, Simons J, Mungall C (2014) Global Biotic Interactions: An open infrastructure to share and analyze species-interaction datasets. Ecological Informatics 24: 148-159. https://doi.org/10.1016/j.ecoinf.2014.08.005

- $\quad$ Ronquist F, Nieves-Aldrey J, Buffington ML, Liu Z, Liljeblad J, Nylander JAA (2015) Phylogeny, evolution and classification of gall wasps: the plot thickens. PLoS One 10 (5): e0123301. https://doi.org/10.1371/journal.pone.0123301

- Weld L (1957) Cynipid galls of the Pacific slope. 1. Privately published, Ann Arbor, MI, USA, 116 pp. URL: https://hdl.handle.net/2027/coo.31924018313555

- Weld L (1959) Cynipid galls of the eastern United States. Privately published, Ann Arbor, MI, USA, 124 pp. URL: https://archive.org/details/cynipidgallseas00weld

- Weld L (1960) Cynipid galls of the southwest. Privately published, Ann Arbor, MI, USA, 35 pp. URL: https://hdl.handle.net/2027/coo.31924018354765

- Wieczorek J, Bloom D, Guralnick R, Blum S, Döring M, Giovanni R, Robertson T, Vieglais D (2012) Darwin Core: an evolving community-developed biodiversity data standard. PLoS One 7 (1): e29715. https://doi.org/10.1371/journal.pone.0029715

\section{Supplementary material}

\section{Suppl. material 1: Catalogue of the Cynipidae of North America doi}

Authors: Nastasi, L. F. and Deans, A. R.

Data type: taxon names, distributions, biotic associations, vernacular names

Brief description: A catalogue of the Aulacideini, Ceroptresini, Diastrophini, Diplolepidini, Phanacidini, and Synergini (Hymenoptera: Cynipidae) of North America, including information about the taxon name history, vernacular names, biotic associations, and geographic regions in which these species occur. The checklist was created using Darwin Core data standard, including Darwin Core Extensions for Taxon Description, Literature References, Vernacular Names, and Species Distribution. A glossary of gall morphology is also included. The data are compressed into a Darwin Core Archive.

Download file $(63.04 \mathrm{~kb})$ 